\title{
XFEL MACHINE PROTECTION SYSTEM (MPS) BASED ON UTCA
}

\author{
S. Karstensen, M. E. Castro Carballo, J. M. Jäger, M. Staack (DESY, Hamburg).
}

Abstract

For the operation of a machine like the $3 \mathrm{~km}$ long linear accelerator XFEL at DESY Hamburg, a safety system keeping the beam from damaging components is obligatory. This machine protection system (MPS) must detect failures of the RF system, magnets, and other critical components in various sections of the XFEL as well as monitor beam and dark current losses, and react in an appropriate way by limiting average beam power, dumping parts of the macro-pulse, or-in the worst case-shutting down the whole accelerator. It has to consider the influence of various machine modes selected by the timing system.

The MPS provides the operators with clear indications of error sources, and offers the possibility to mask any input channel to facilitate the operation of the machine. In addition, redundant installation of critical MPS components will help to avoid unnecessary downtime. This document summarizes the requirements on the machine protection system and includes plans for its architecture and for needed hardware components.

\section{INTRODUCTION}

The European X-Ray Free Electron Laser (XFEL) linear accelerator will bring an electron beam to the energy of up to $20 \mathrm{GeV}$ and use it to generate extremely brilliant pulses of spatially coherent $\mathrm{x}$-rays in an array of undulators using the Self-Amplified Spontaneous Emission (SASE) process. With a designated average beam power of $600 \mathrm{~kW}$ and beam spot sizes down to few micrometres, the machine will hold a serious damage potential. To ensure safe operation of the accelerator, it is therefore necessary to detect dangerous situations by closely monitoring beam losses and the status of critical components, and to react appropriately. This is the task of the fast machine protection system (MPS) described in this paper. Several design features of the system have been influenced by experience from existing facilities, particularly the Free Electron Laser in Hamburg (FLASH).

A high flexibility of the MPS is essential to guarantee minimum downtime of the accelerator. In contrast to a storage ring where a beam dump typically implies a timeconsuming refill of the machine, a linac offers the possibility to limit the length of the bunch train individually for each macro-pulse. Hence the reaction to failures of subsystems or even parts of the MPS can be much more specific - a dynamic limitation of the total beam power or a selective veto on beam transport into a particular branch of the beamline are possible. Experience with FLASH has also shown that the operation of the machine profits from an MPS whose behaviour can be changed or extended in a simple way.

\section{XFEL ARCHITECTURE}

The present chapter provides a brief overview of the XFEL facility (Figure 1). The major tunnel sections accommodate the following systems:

- injector

- linear accelerator (linac)

- beam distribution system

- undulators

- photon beam lines

- experimental stations

These components are distributed along an essentially linear geometry, $3.4 \mathrm{~km}$ long, starting on the Deutsches Elektronen-Synchrotron (DESY) laboratory campus in the north west part of the city of Hamburg, and ending in the neighbouring federal state of Schleswig-Holstein, south of the city of Schenefeld, where the experimental hall will be located.

In the injector, electron bunches are extracted from a photocathode by a laser beam, focused and accelerated in a radio frequency (RF) gun and a superconducting acceleration module, and directed towards the linac with an exit energy of $120 \mathrm{MeV}$. After further acceleration, the bunches are longitudinally compressed in two bunch compressors, $\mathrm{BC} 1$ and $\mathrm{BC} 2$, at energies of $500 \mathrm{MeV}$ and $2 \mathrm{GeV}$. In the adjacent main linac, the beam is brought to energies of up to $20 \mathrm{GeV}(17.5 \mathrm{GeV}$ is the energy foreseen for normal operation of the XFEL) before passing the collimation section. Afterwards, a fast kicker can send single bunches into a beam dump. The remaining bunch train can be sent into two undulator lines by the beam distribution kicker with a rise time of less than $20 \mu \mathrm{s}$. Each of the undulator lines ends in an electron beam dump, and each of the three main beam dumps is designed to withstand only half of the nominal beam power, i.e. $300 \mathrm{~kW}$.

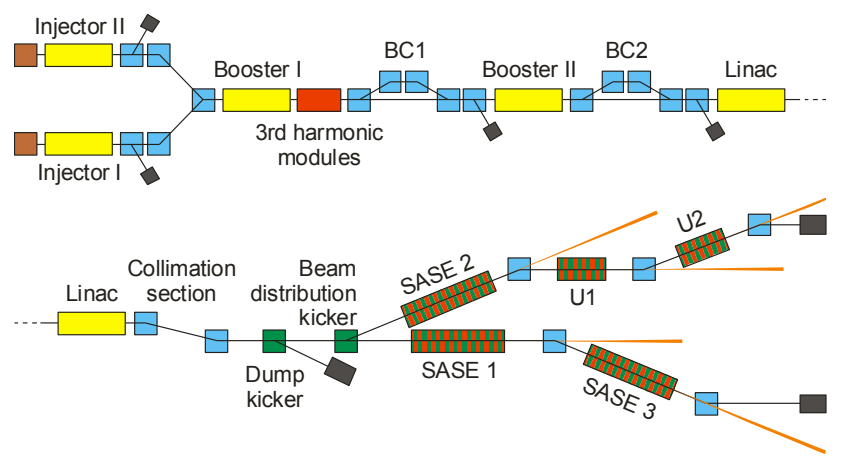

Figure 1: Schematic of the XFEL beam line 


\section{REQUIREMENTS FOR THE MACHINE PROTECTION SYSTEM}

We can summarize the main requirements for the MPS in three points, in the order of their importance:

1. protect accelerator components and devices in the vicinity of the accelerator from direct and indirect damage by the beam

2. facilitate easy handling of the machine-impair machine operation only if necessary

3. limit activation of accelerator components to preserve their maintainability

While it is obvious that the protection from damage is of paramount priority, any machine can only serve its purpose if it can be operated. Beam time at the XFEL will be in high demand, and the goal should be to limit downtimes to their necessary extent. In this respect, it is of no importance whether downtimes are caused by hardware failures or operating errors; the MPS should be both highly reliable and "user-friendly".

\section{Failsafe behavior:}

Since most of the MPS electronics will be located in the accelerator tunnel, an elevated radiation background must be expected. An analysis on the behavior of FPGAs has been carried out at DESY [1] and shows that neutroninduced single event upsets (SEUs) are the major source of malfunctions. Therefore, the design of the electronics should ensure as far as possible that SEUs do not lead to unsafe or uncontrolled behavior of the system. Problems caused by power cuts or simple cable breaks must also be considered.

\section{Reaction times:}

In the XFEL, the distance from the injector lasers to the last undulators is approximately $3 \mathrm{~km}$. Thus, at the speed of light, a signal needs about $10 \mu$ s to travel from one end to the other. Since the bunch frequency of the XFEL is $5 \mathrm{MHz}$, a maximum of 50 bunches are moving within the accelerator at any given time.

Table 1: Minimum reaction times to MPS alarms at various locations, assuming a signal velocity of $2 / 3 \mathrm{c}$.

\begin{tabular}{|l|r|r|r|}
\hline $\begin{array}{l}\text { Beam loss } \\
\text { location }\end{array}$ & $\begin{array}{l}\text { Distance } \\
\text { from } \\
\text { injector }\end{array}$ & $\begin{array}{l}\text { Distance } \\
\text { from dump } \\
\text { kicker }\end{array}$ & $\begin{array}{l}\text { min. num. } \\
\text { of lost } \\
\text { bunches }\end{array}$ \\
\hline Injector & $0 \mathrm{~m}$ & $-1970 \mathrm{~m}$ & 0 \\
\hline bunch compr. 1 & $160 \mathrm{~m}$ & $-1810 \mathrm{~m}$ & 7 \\
\hline bunch compr. 2 & $360 \mathrm{~m}$ & $-1610 \mathrm{~m}$ & 15 \\
\hline linac center & $1040 \mathrm{~m}$ & $-930 \mathrm{~m}$ & 44 \\
\hline linac end & $1650 \mathrm{~m}$ & $-320 \mathrm{~m}$ & 69 \\
\hline $\begin{array}{l}\text { beam distribution } \\
\text { kicker }\end{array}$ & $2010 \mathrm{~m}$ & $40 \mathrm{~m}$ & 2 \\
\hline last undulator & $3010 \mathrm{~m}$ & $1040 \mathrm{~m}$ & 44 \\
\hline
\end{tabular}

From this follows that, if beam losses are detected near the end of the machine, at least 100 bunches carrying a total energy of about $2.2 \mathrm{~kJ}$ would arrive at the position of the loss before the injector laser could be switched off.

To improve this reaction time, the MPS clearly needs a second location for stopping the beam. The dump kicker, part of the beam switchyard at about $2.1 \mathrm{~km}$ along the machine, is the natural choice for this interaction. Table 1 lists the minimum possible reaction times and the minimum number of lost bunches at several possible locations of MPS alarms.

\section{MPS ARCHITECTURE}

The large scale of the XFEL imposes a severe technical issue: latency of electronics and signal transport speed of $3 / 4 c$ in copper cables and $2 / 3 c$ in optical fibers lead to a signal delay and in consequence additional lost bunches. To provide a short reaction time, the MPS implements a distributed Master/Slave architecture keeping short distances between components.

All RS422 [2] input signals are handled equally fast. There is no difference between fast and slow incoming system signals. Also all signals were processed with the fastest speed of the FPGA.

The optical fibers are planned in a way that a set of fibers connects each RF section (4 cryogenic modules, 1 klystron) to the injector building XSE. Various points in the undulator sections are connected to the hall XS1, fiber sets from the experimental stations are collected in the hall XHEXP1. There are fiber connections between XSE, XS1 and XHEXP1. Each of the RF sections will be equipped with 2 MPS modules ( Figure 1). Loops in the bunch compressors and SASE sections will contain 3 to 5 MPS modules.

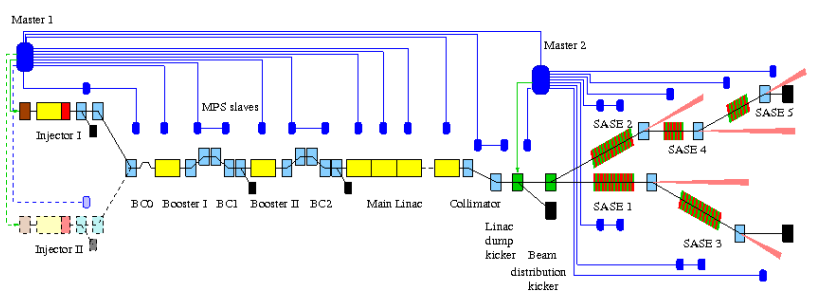

Figure 1:MPS distributed in XFEL

The backbone of the XFEL Machine Protection System consists of roughly 130 MPS slave modules distributed along the machine. Each of these modules has digital inputs for "beam OK" signals from critical subsystems, accelerator components, or beam loss detection hardware. It also has fast serial input and output ports to connect to other MPS modules; to avoid problems with electromagnetic interference, these serial connections use fibre optic cables.

All modules are developed to provide high flexibility. They could be used as an intelligent data distribution knot with more or less intelligence or just as a simple data collector, with single or redundant communication links.

Two master modules are located near the injector and near the linac dump kicker (Figure 2). The FPGA logic of these "MPS masters" defines the behavior of the system. They have direct connections to the injector lasers and to 
the dump kicker, allowing them to stop the production of new bunches and to dump bunches that are already in the machine. Both Masters are working very close together and can be seen as one Master. The latency between the two masters is negligible, as both masters have different tasks and the data stream between both is only informal.

Referring to the XFEL Timing System [3], the complete MPS system is working asynchronously.

The serial connections between MPS masters and slaves form loops carrying a steady data stream. Within reasonable limits, the number of loops connected to each master can be chosen freely, while the number of slave modules in each loop is limited mainly by the desired latency of less than $1 \mu$ s excluding cable delays.

Simulations have shown that with current FPGA technology, the input latency per board is in the range of 40 ns (RS 422 input $\rightarrow$ FPGA).

The hardware allows different topologies, which will be discussed briefly in the following sections.

The topology of the MPS is a mixture of daisy chain and star topology to balance their benefits and drawbacks.

The daisy chain topology reduces the cabling and serial connection to the master but increases the latency and makes every module a single point of failure for the whole system. On the contrary, the star topology, with a direct connection between each slave and the master, reduces the latency and allows ignoring single modules in case of error. The drawback of the star topology is that it is the most expensive option.

For the XFEL, the chosen mixed topology allows to set up small loops of few modules ( Figure 2) that are arranged so that the failure of one loop would only compromise few important signals; in addition, critical modules can be made redundant. The system would also be easily scalable without major cabling work because new slaves could be added to existing loops.

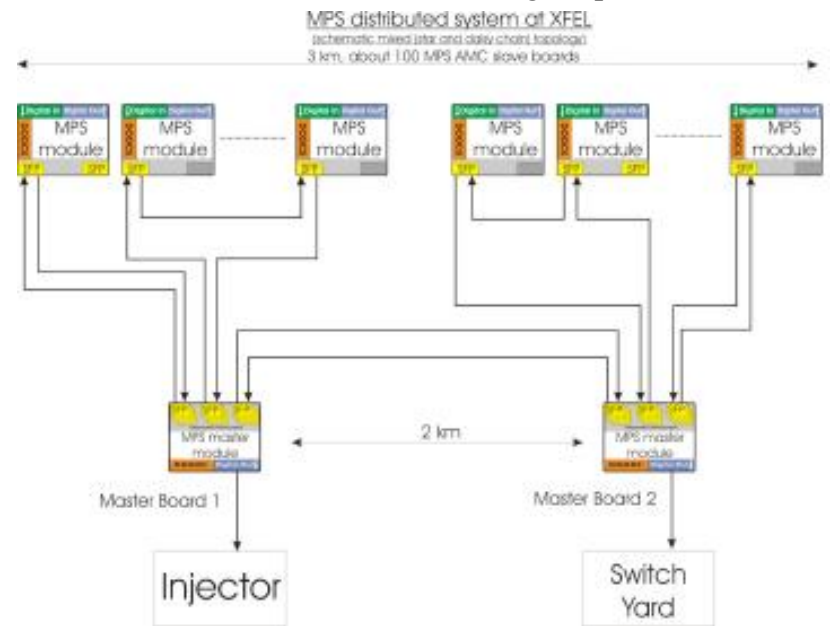

Figure 2: MPS distributed system schematic

\section{INTERFACES}

The MPS slaves receive digital status signals from external systems via RTM / RS422 signal lines and information from the Timing System via the $\mu$ TCA backplane. A cable break or short circuit within the
RS422 lines will be detected and reported as an alarm without distinguishing between real alarm or cable break.

The main MPS master is connected to the Timing System to provide the information about possible Beam Modes (Figure 3) and Section patterns ( Figure 4). Also, since MPS slaves and masters constantly communicate with each other, this communication is watched by special algorithms to guaranty a failsafe operation.

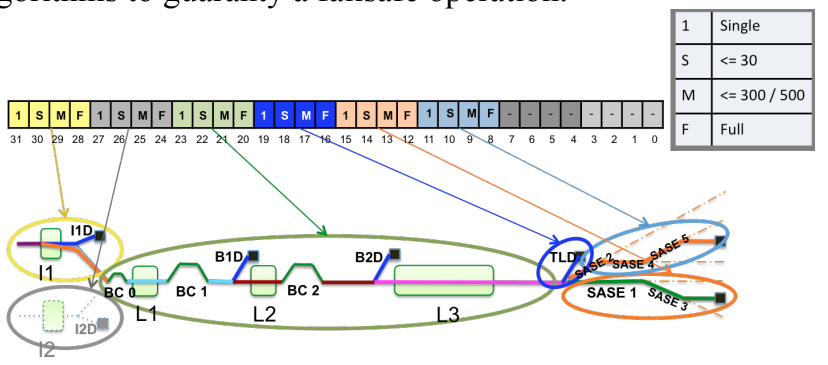

Figure 3: XFEL Beam Modes

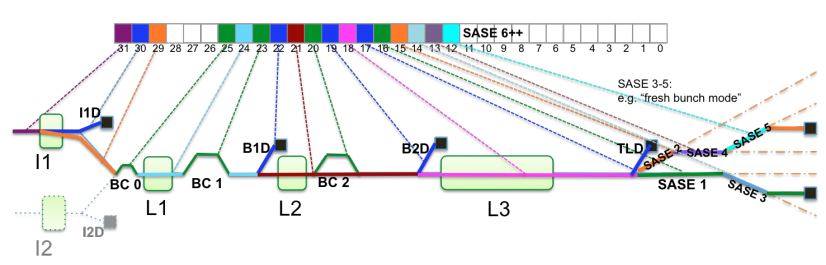

Figure 4: XFEL Section Patterns

All connected systems are shown in Figure 5. All incoming signals are handled equally. There is no difference between slow (e.g. relay) or fast (e.g. electronic) signals. The "Speed" column in the table is only for background information (time between event and sending an alarm to MPS).

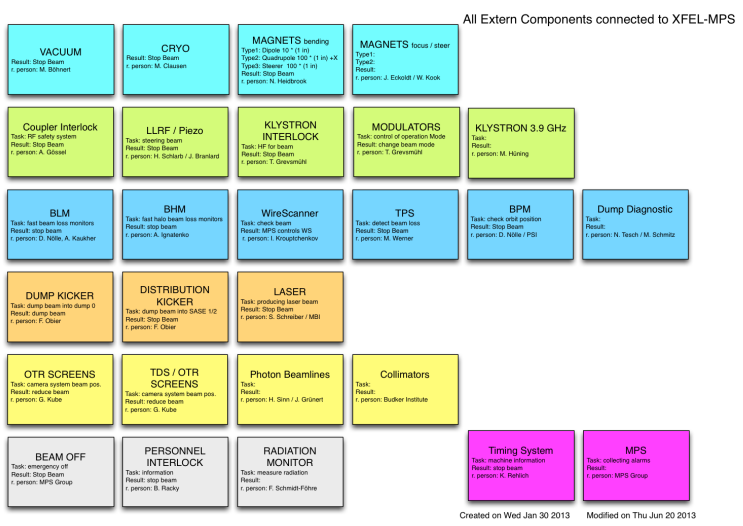

Figure 5: Connected systems to MPS

\section{MTCA TECHNOLOGY}

MPS as same as most of the control components of XFEL will use the $\mu$ TCA technology as of the following reasons:

- Modular + modern architecture

- $\quad$ Reusability + PCIe + Ethernet

- High availability

- Redundant power and fan optional

- Well defined management 
- High performance:

- Very low analog distortions

○ 4 lanes PCIe: $400 \mathrm{MB} / \mathrm{s}$... $3.2 \mathrm{~GB} / \mathrm{s}$

XFEL fast electronics will be based on MTCA.4: with more than 200 Crates [4]

\section{HARDWARE}

The Master and Slave boards are exclusively equipped with the DESY DAMC2 board. This strategy offers a large flexibility in case of technical problems as the DAMC2 is used everywhere inside the XFEL and MPS will take profit out of external developments, soft- and hardware wise. To receive digital alarm signals at DAMC2, a Rear Transition Module (RTM) has been developed for MPS. A schematic diagram is shown in Figure 6.

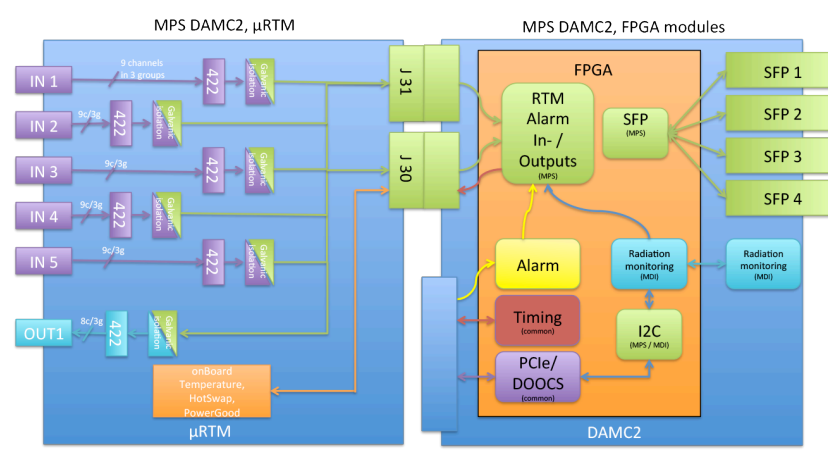

Figure 6: DAMC2 and RTM structure

\section{DAMC2}

The DAMC2 board of Figure 7 has been developed by DESY and is equipped with a VIRTEX 5 FPGA. The documentation is available at DESY FEA group.

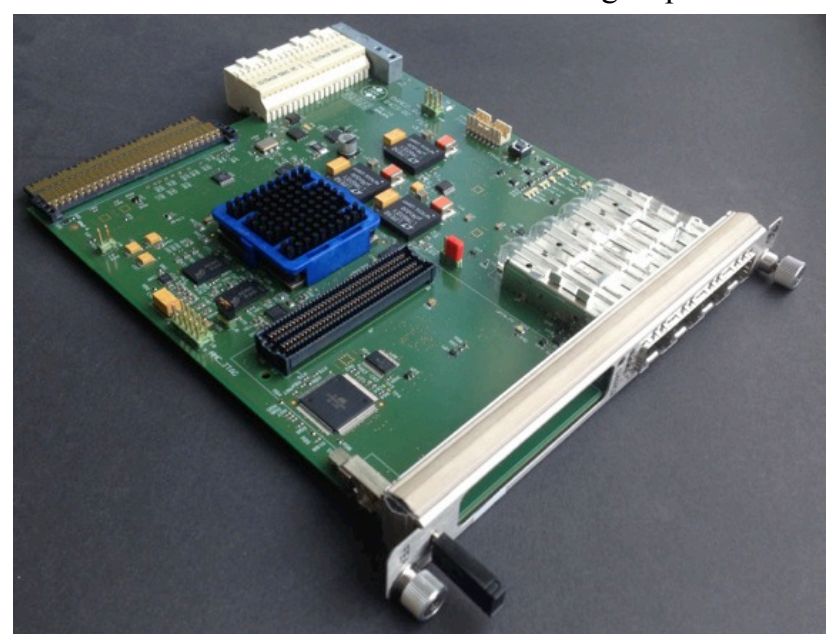

Figure 7: DAMC2 board

\section{MPS RTM}

The MPS RTM, shown in Figure 8, is the interface between RS422 in- and outputs and the DAMC2 board. It is developed at DESY and fulfills all requirements to be used with the DAMC2. The latency of an input signal from RTM to the FPGA of the DAMC2 has been measured with 42 ns per channel [5]. See also Figure 9 for more latency info. This has been measured in August 2013 and will be approved .

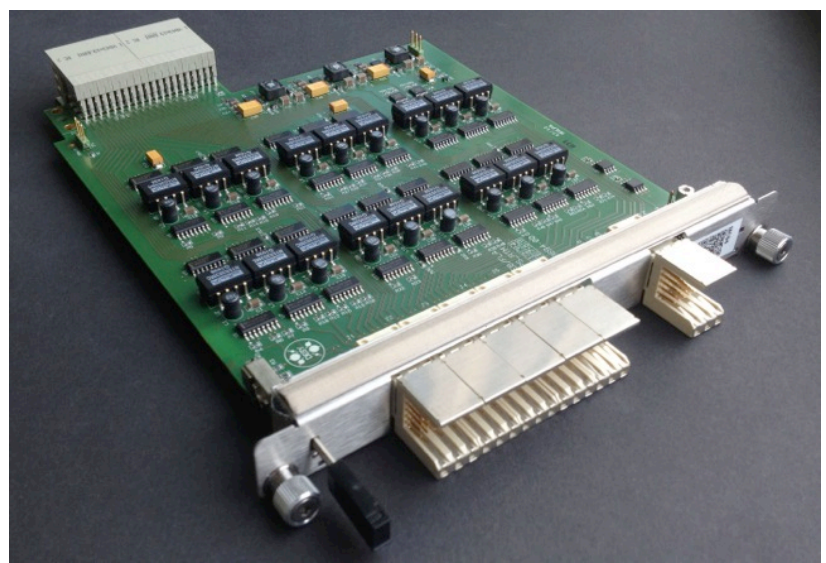

Figure 8: MPS RTM module

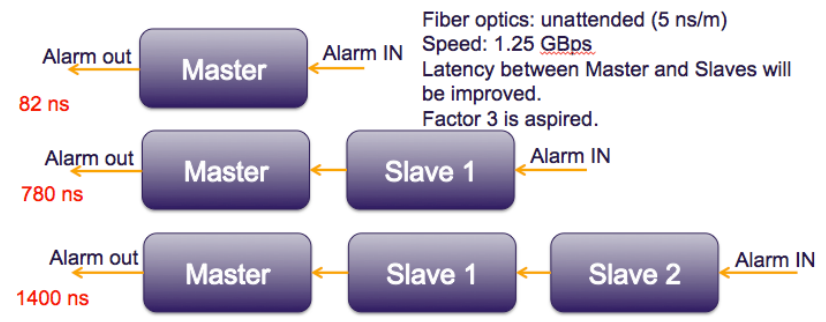

Figure 9: Latency measurement of several combinations of MPS modules

\section{GLOSSARY}

Macro-pulse A shot in the linac of a train of electron bunches with duration of up to $\sim 600 \mu \mathrm{s}$ plus the filling and decay time of the cavities. Usually repeated every $10 \mathrm{~Hz}$

Beam Mode It defines the maximum number of bunches permitted per macro-pulse in a section of the accelerator. The MPS grants the mode by reading the machine settings and interlocks.

Section Pattern Every bit in the pattern describes the status of a subsection. If the bit is set to ' 1 ', beam is permitted within this subsection

\section{REFERENCES}

[1] D. K. Rybka, et al., Irradiation investigations for TESLA and X-FEL experiments at DESY. Report TESLA 2004-12, DESY, 2004.

[2] RS422 and RS485 Standards Overview and System http://focus.ti.com/lit/an/slla070d/slla070d.pdf

[3] A. Aghababyan, C. Bohm, P. Gessler, A. Hidvégi, H. Kay, G. Petrosyan, L. Petrosyan, V. Petrosyan, K. Rehlich, XFEL Timing System Specifications Version 1.0, 3. 12. 2012

[4] Kay Rehlich, ARD Workshop, 23.08.2012

[5] S. Karstensen, Function and Latency test at MPSRTM, September 2012 